\title{
Osteogenic effect in rat osteoporosis model and femur defect model by intraosseous administration of simvastatin microcrystals
}

Junxiong Zhu

Peking University Third Hospital

Chenggui Zhang

Peking University Third Hospital

Jialin Jia

Peking University Third Hospital

Hong Wang

Peking University Third Hospital

Huijie Leng

Peking University Third Hospital

\section{Yingsheng Xu}

Peking University Third Hospital

Cuishuan Wu

Beijing Delivery Pharm Technology Co

Qiang Zhang

Peking University

Chunli Song ( $\square$ schl@bjmu.edu.cn )

Peking University Third Hospital

\section{Research}

Keywords: simvastatin, microcrystal, osteoporosis, bone defect, hydrogel, calcium phosphate cement

Posted Date: June 8th, 2020

DOl: https://doi.org/10.21203/rs.3.rs-32323/v1

License: (c) (i) This work is licensed under a Creative Commons Attribution 4.0 International License.

Read Full License 
Osteogenic effect in rat osteoporosis model and femur defect model by intraosseous administration of simvastatin microcrystals

\author{
Junxiong Zhu, ${ }^{1}$ Chenggui Zhang, ${ }^{1}$ Jialin Jia, ${ }^{1}$ Hong Wang,${ }^{1,2}$ Huijie Leng, ${ }^{1,2}$ Yingsheng $\mathrm{Xu},{ }^{3}$ Cuishuan $\mathrm{Wu},{ }^{4}$ \\ Qiang Zhang, ${ }^{5}$ and Chunli Song ${ }^{1,2}$ \\ Correspondence should be addressed to Chunli Song (schl@bjmu.edu.cn).
}

\begin{abstract}
Background: Currently, drugs for local bone formation are very limited in clinic. Simvastatin is one of most potential translational drugs by repositioning for its significant osteogenic effect and its history as a safe lipid-lowering medicine. In this study, we prepared the pre-clinical simvastatin microcrystals which could be potentially translated and industrialized.
\end{abstract}

Methods: Simvastatin microcrystals were made by wet media milling method. Characterization of microcrystal particles were evaluated by laser particle size analyzer and high-performance liquid chromatography (HPLC). Furthermore, we verified the osteogenic effect in rat ovariectomy(OVX)-induced osteoporosis model and femur defect model. For osteoporosis model, we delivered simvastatin microcrystals to tibia with poloxamer hydrogel by intraosseous injection. Bone mineral density (BMD) and ultimate force were assessed after treatment. For femur defect model, simvastatin microcrystal was incorporated in clinically used calcium phosphate cements (CPCs) as an implant. Histology and $\mu \mathrm{CT}$ were used for evaluation of the healing.

Results: The $\mathrm{D}_{10}, \mathrm{D}_{50}$, and $\mathrm{D}_{90}$ showed size of the most particles ranged from 0.226-3.425 $\mu \mathrm{m}$. The mean particle size of the microcrystals is determined as $1.365 \mu \mathrm{m}$ which demonstrated a successful preparation. For osteoporosis model, BMD and the ultimate force of the treated tibia were significantly improved in osteoporosis rat after injection of $0.5 \mathrm{mg}$ and $1 \mathrm{mg}$ simvastatin microcrystals compared with OVX or $0 \mathrm{mg}$ groups. There were no differences observed in BMD and ultimate force between $0.5 \mathrm{mg}, 1 \mathrm{mg}$ simvastatin microcrystals group and bone morphogenetic protein (BMP) 5 ug group. For femur defect model, quantitative analysis of bone regeneration by $\mu \mathrm{CT}$ showed bone volume/tissue volume (BV/TV), trabecular thickness (Tb.Th), trabecular number (Tb.N) were significantly increased in simvastatin microcrystals 50ug (SIM-C 50 ug), SIM-C 100 ug and BMP 5 ug group compared with CPC vehicle group. Trabecular separation (Tb.Sp), and cement volume/tissue volume (CV/TV) were decreased in SIM-C 50 ug, SIM-C 100 ug and BMP 5 ug groups compared with CPC vehicle group. Semiquantitative scale for histology assessment further demonstrated a higher bone regeneration score in drug loaded groups compared with CPC group.

Conclusions: Our study shows that simvastatin microcrystals were successfully prepared by wet media milling method and the microcrystals can promote bone formation by local delivery by poloxamer hydrogel or CPC, which is of great translational potential.

Keywords: simvastatin, microcrystal, osteoporosis, bone defect, hydrogel, calcium phosphate cement 


\section{Background}

The healing of bone defects remains a significant clinical orthopedic challenge. Autologous bone grafts are considered as the gold standard. However, these procedures may cause donor-site morbidity, such as hemorrhage, infection, and chronic pain.[1] With the general aging of the population, osteoporosis is a burgeoning public health problem of global concern. However, the ultimate goal of osteoporosis treatment is the prevention of the initial and subsequent occurrences of fragile fractures. Local treatment strategy targeting specific bone regions that are prone to fractures may help to decrease the incidence of osteoporotic fractures. Both prevention of osteoporotic fracture and improvement of bone defect healing could be achieved by locally application of osteogenic drugs.

Statins, which are inhibitors of HMG-CoA (3-hydroxy-3-methylglutaryl-coenyzyme A) reductase, have been used for many years to treat hyperlipidemia and to prevent cardiovascular events. Since Mundy et al. first reported on the anabolic effects of statins on bone formation,[2] emerging pre-clinical studies have demonstrated the ability in promoting bone formation in different in vitro and in vivo studies.[3] However, controversies still remain due to low bioavailability of orally administered statins to the skeleton.[4] After the first-pass hepatic metabolism, only approximately $5 \%$ of the active compound remains in the circulation, even less at the defect site.[5] Increasing the dosage is not an option, as high-dose administration of statins may cause adverse side effects. Local delivery of simvastatin is another option that can be used. Our previous studies have shown locally applied statins with sustained release can improve the quality of osteoporotic bone, promote defect healing or enhance pedicle-screw fixation in animals from rats[6], rabbits[7] to minipigs[8,9] and rhesus macaques (unpublished data).

Although these results are promising, the current method for simvastatin delivery in pre-clinical studies is impractical for human application and may impede its clinical translation. Simvastatin is a lipophilic drug that is difficult to dissolve in water. Low aqueous solubility of active pharmaceutical ingredients is a major problem in the design of pharmaceutical.[10] When mixing simvastatin with hydrophilic hydrogel, it's difficult to ensure the homogeneous distribution of drug in vehicle, the large drug particles may cause a precipitation during storage and even application, and also the drug release may not be controlled, due to the heterogeneous particle sizes of drug raw materials. Our study also found that simvastatin in poloxamer hydrogel is not stable and the ${ }^{60} \mathrm{Co}$ irradiation for sterilization can accelerate its degradation rate.

Pharmaceutical formulation is the process in which different chemical substances, including the active drug, are combined to produce a final medicinal product. Thus, better pharmaceutical preparation process with industrialization and translation benefits is also needed.

As one of the sustained-release drug delivery systems, microcrystals have been increasingly exploited for poorly water-soluble drugs. Microcrystals has a small particle size (1 to $100 \mu \mathrm{m}$ ), a high drug loadings (the drug content can be close to $100 \%$ ), and are suitable for multiple administration routes and easy to industrial production. The homogeneous particle sizes of microcrystals result in well-controlled drug release, improved physical stability in vehicle, as well as dose homogeneity, besides their long-time therapeutic effect following a single injection.[11] Based on these reasons, here we produced simvastatin microcrystals for intraosseous application by new formulation and preparation method, hoping to achieve an uniform osteogenesis effect which is favorable for its clinical use. 
For easy translation, two well-studied delivery methods with according animal models were used to test the osteogenic effect of simvastatin microcrystals. The first is Poloxamer hydrogel as it demonstrates biocompatibility, low toxicity, and weak immunogenicity. It also demonstrates reversible thermosensitive gelation (with the "sol-gel" characteristics of being a liquid at low temperature but a semisolid gel at body temperature), which makes it suitable as a vehicle for drug delivery.[12, 13] Among them, Poloxamer 407 is an excipient of various formulations that is approved by the U.S. Food and Drug Administration for pharmaceutical application.[14] The other is calcium phosphate cements (CPCs). CPCs have long been used as osteoconductive bone substitutes in the treatment of bone defects. Calcium phosphate cement consists of one or more calcium orthophosphate powders that are mixed with an aqueous solution. The paste that forms will harden within a few minutes to a solid body. Clinically, antibiotics or bone morphogenic proteins can be added to the CPCs to treat infectious disease or improve bone healing. This study is aimed to test whether simvastatin microcrystals could remain ontogenetic effect delivered by poloxamer or CPC to promote local osteogenesis.

\section{Materials and Methods}

Experiment 1: preparing simvastatin microcrystals by wet media milling method

\section{Study design}

The pre-clinical simvastatin microcrystals were prepared through wet media milling method. The lyophilized sample was re-dissolved by $1 \mathrm{~mL}$ of sodium chloride injection then undergoes sonication (in a water bath). Further particle size determination was then implemented. The moisture and impurities were also determined.

\section{Preparation of simvastatin microcrystals}

A solution of Tween 80 in sterile water for injection was prepared under stirring until its complete dissolution. Simvastatin was added to Tween 80 solution and agitated for $1 \mathrm{~h}$ to produce the simvastatin suspension. Then, the obtained simvastatin suspension was transferred to the grinding miller and grinded for $1 \mathrm{~h}$ at $3000 \mathrm{rpm}$. Mannitol was added to the grinding solution and stirred to dissolve. The resultant solution was diluted to the corresponding concentration with sterile water for injection. The finished product was obtained by freeze-drying from the solution. Table 1 shows the preparation of simvastatin microcrystals.

Table 1 Final formulation and preparation process for production of simvastatin microcrystals.

\begin{tabular}{ll}
\hline Raw material & Dosage $(\mathrm{mg} /$ ampoule $)$ \\
\hline Simvastatin & 1 \\
Tween 80 & 0.3 \\
Mannitol & 50 \\
Micronization times & 3
\end{tabular}


Freeze-drying process and parameters: the freezing temperature: $-40^{\circ} \mathrm{C}$; the freezing time: $300 \mathrm{~min}$; primary drying: $-20^{\circ} \mathrm{C}-150 \mathrm{~min} ;-8^{\circ} \mathrm{C}-240 \mathrm{~min}-210 \mathrm{~min} ;-6^{\circ} \mathrm{C}-120 \mathrm{~min}-300 \mathrm{~min} ; 0^{\circ} \mathrm{C}-180 \mathrm{~min}-0 \mathrm{~min}$, ; secondary drying: $10^{\circ} \mathrm{C}-90 \mathrm{~min}-0 \mathrm{~min} ; 20^{\circ} \mathrm{C}-120 \mathrm{~min}-0 \mathrm{~min}$.

\section{Particle size determination}

The particle size and distribution was measured by a laser particle size analyzer (BT-9300S, Bettersize Co., Ltd., Dandong, China). $1 \mathrm{~mL}$ of sodium chloride injection was added to the simvastatin microcrystals and sonicated (in a water bath) for $2 \mathrm{~min}$. The distilled water was added into $2 / 3$ of the cuvette for background calibration. 3-5 drops of the prepared sample solution was added into the sizing cuvette and stirred for $30 \mathrm{~s}$. The sample was analyzed for 3 times. The $\mathrm{D}_{10}, \mathrm{D}_{50}$, and $\mathrm{D}_{90}$ as well as the obtained span were correlated with $10 \%, 50 \%$, and $90 \%$ of the cumulative particle size distribution.

\section{Determination of moisture and impurities}

The water content was determined by moisture determination method according to the Pharmacopoeia of the People's Republic of China Four General Rules 0832 (2015 Edition). To determine the related substance of simvastatin microcrystals, high-performance liquid chromatography (HPLC) using SUPELCO LC-C18-DB column $(3.3 \mathrm{~cm} \times 4.6 \mathrm{~mm})$ containing particles measuring $3 \mu \mathrm{m}$ in diameter at $35^{\circ} \mathrm{C}$ was used. The ultraviolet wavelength was $238 \mathrm{~nm}$. The mobile phase A was acetonitrile $/ 0.1 \%$ phosphoric acid solution $(50 / 50, \mathrm{v} / \mathrm{v})$ and the mobile $B$ was acetonitrile containing $0.1 \%$ phosphoric acid at a flow rate of $3.0 \mathrm{~mL} / \mathrm{min}$. The gradient elution program was shown in the below table 2 . The injection volume was $10 \mu \mathrm{L}$ and the run time was 13 $\mathrm{min}$. To achieve a final concentration simvastatin of $0.8 \mathrm{mg} / \mathrm{ml}$, the simvastatin microcrystals were dissolved in the $\mathrm{pH} 4.0$ solvent containing acetonitrile and $0.01 \mathrm{~mol} / \mathrm{L}$ potassium dihydrogen phosphate solution $(3: 2$, $\mathrm{v} / \mathrm{v})$. The test solution was obtained by filtered using $0.22 \mu \mathrm{m}$ microfiltration membrane. The reference solution was made by accurately pipetting $1.0 \mathrm{~mL}$ of the test solution into a $100 \mathrm{~mL}$ volumetric flask and diluting to volume with the $\mathrm{pH} 4.0$ solvent.

Table 2. Gradient elution

\begin{tabular}{lll}
\hline Time $(\min )$ & Mobile phase A $(\%)$ & Mobile phase B $(\%)$ \\
\hline 0 & 100 & 0 \\
\hline 4.5 & 100 & 0 \\
\hline 4.6 & 95 & 5 \\
\hline 8.0 & 25 & 75 \\
\hline 11.5 & 25 & 75 \\
\hline 11.6 & 100 & 0 \\
\hline 13 & 100 & 0 \\
\hline
\end{tabular}




\section{Sterilization of simvastatin microcrystal}

The drug substance and excipients are sterilized in the manufacturing process. The manufacturing plant, the vial, rubber stopper and aluminum cap are sterilized. Therefore, there is no special sterilization method for the final microcrystals.

Experiment 2: verifying the osteogenic effect of simvastatin microcrystals with hydrogel in osteoporosis rat model

\section{Study design}

After 2 weeks of acclimation, eighty four 3-month-old Sprague-Dawley female rats were randomly divided into 7 groups ( $\mathrm{n}=12$ rats /group): 1 . Sham (without removing ovary after exposing and without intraosseous injection); 2. OVX (ovariectomy) group without intraosseous injection as control; 3. SIM-C (simvastatin microcrystals) $0 \mathrm{mg}$ as vehicle control (accessory only) ; 4. SIM-C $0.5 \mathrm{mg} ; 5$. SIM-C $1 \mathrm{mg}$; 6. SIM-BPC (simvastatin bulk pharmaceutical chemicals which are the raw material for microcrystals, provided by Zhejiang Jiangbei Pharmaceutical Co., Ltd. ) $0.5 \mathrm{mg}$; 7) BMP (rhBMP-2, provided by Shanghai Rebone Biomaterials Co., Ltd.) 5 ug. Rats received bilateral OVX to induce osteoporosis under isoflurane, as described previously.[15] Three months later after OVX the animals will demonstrated osteoporosis and receive the intraosseous injection. A month later after the intraosseous injection, the animals were sacrificed for further assessment.

\section{Simvastatin microcrystals in Poloxamer 407 Gel Formulation}

Poloxamer 407 (25\% w/w; BASF) was added to isotonic phosphate-buffered saline (PBS) solution (pH 7.4, $4^{\circ} \mathrm{C}$ ) with gentle mixing until it dissolved as previously described.[8] SIM-C, SIM-BPC or BMP in poloxamer 407 gels were prepared by adding them to the poloxamer 407 solution. The final simvastatin concentrations were 0,5 , and $10 \mathrm{mg} / \mathrm{ml}$. Concentration of BMP was $50 \mathrm{ug} / \mathrm{ml}$.

\section{Local intraosseous injection of simvastatin microcrystals / Poloxamer 407 gel}

We located the entry point at the front of the tibial intercondylar eminence and inserted the needle parallel to the long axis of the tibia as previously describe.[16] A tibial radiograph ensured that the needle tip was in the medullary cavity (this validation step can be omitted after sufficient procedural skill is gained) and $100 \mathrm{ul}$ solution was given to the right tibia marrow cavity for each animal.

\section{BMD}

Bone mineral density (BMD) was measured using dual-energy X-ray absorptiometry (DXA) for smallanimal high-resolution (UltraFocus DXA, Faxitron). The region of interest (ROI) of BMD was set to the whole bone.

\section{Biomechanical test}

A three-point bending test was used to evaluate the mechanical properties of tibia using a mechanical testing system (MTS Landmark Systems, Eden Prairie, MN, USA) with a load cell of 100 N. Before testing, samples were thawed at room temperature for $1 \mathrm{~h}$. Tibias were placed with their posterior side 
facing down between two supports with a $15-\mathrm{mm}$ span. A preload of $0.5 \mathrm{~N}$ was applied at the middle point of the bone to hold it in position. A compressive force was applied to the bone at a speed of $1 \mathrm{~mm} / \mathrm{min}$. Maximum bending force was calculated based on the resulting force-displacement curves.

\section{Experiment 3: verifying the osteogenesis effect of simvastatin microcrystals with $\mathrm{CPC}$ in bone defect rat model}

\section{Study design}

Four groups, the CPC group, CPC+BMP 5ug, CPC+SIM-C 50 ug and CPC+SIM-C 100 ug were designated, with 10 rats in each group. Under anesthesia by isoflurane, a longitudinal incision was made to expose the metaphyseal region of the distal femur. A bone defect of $3 \mathrm{~mm}$ of diameter and $5 \mathrm{~mm}$ of length was created as previously described.[17] A clinically available CPC (Shanghai Rebone Biomaterials) was used to create cylindrical samples with a $3 \mathrm{~mm}$ of diameter and $5 \mathrm{~mm}$ of height were prepared. Simvastatin microcrystals or rhBMP-2 (Shanghai Rebone Biomaterials) powder was mixed with liquid phase in different ration before adding the curing liquid to solid phase. The final simvastatin concentration is 7.2 or $14.4 \mathrm{mg} / \mathrm{ml}$ and BMP concentration is $0.72 \mathrm{mg} / \mathrm{ml}$. In this way each cylindrical CPC will contain around 50 ug, 100ug simvastatin and 5 ug BMP by calculating the volume of cylindrical samples. The bone defect was then reconstructed with cylindrical samples with different doses of drugs (Fig. 2A). The surgical wound was then closed in layers. Animals were sacrificed 8 weeks after the operation for therapeutic assessment.

\section{SEM}

The fresh fractured surfaces of the cements were examined under scanning electron microscopy (FE-SEM, S-4800, Hitachi, Japan). The samples were sputter coated with a 20 -nm-thick layer of gold prior to examination.

\section{Serum assay}

Whole blood was collected by cardiac puncture at euthanasia and subsequently centrifuged at $2500 \times \mathrm{g}$ for $20 \mathrm{~min}$ at $4^{\circ} \mathrm{C}$ to separate serum for measurement of serum bone formation marker, intact $\mathrm{N}$-terminal propeptide of type-1 procollagen (P1NP) and bone resorption marker, tartrate-resistant acid phosphatase $5 \mathrm{~b}$ (TRACP 5b) by enzyme-linked immunosorbent assay (Shanghai Jianglai Biotech, Shanghai, China).

\section{Histology}

Histologic examinations were performed to verify the situation of the implant and bone tissue ingrowth. For the decalcification protocol, the specimens were decalcified by $10 \%$ citric acid after fixation and then dehydrated in a graded ethanol series (ethanol, 90, 95, and 100\%). The specimens were then embedded in paraffin and sectioned to a thickness of $6 \mathrm{~mm}$ in an orientation that was parallel to the long axis of the bone defect. The sections were stained by Hematoxylin and Eosin or Masson's trichrome and examined under a light microscope.

\section{$\mu \mathrm{CT}$}

Specimens were scanned by $\mu \mathrm{CT}$ (Inveon, Siemens, Erlangen, Germany) at an effective pixel size of 8.82 
$\mu \mathrm{m}, 80 \mathrm{kV} / 500 \mu \mathrm{A}$, and a 1500-ms exposure time in each of the 360 rotational steps. Cylindrical CPC degradation and new bone regeneration were observed. A standard cylindrical (3 mm diameter $\times 5 \mathrm{~mm}$ height) volume of interest (VOI) was created at the location where the CPC were implanted. Within this standardized VOI, bone regeneration indices were examined including bone volume (BV), cement volume (CV), trabecular thickness (Tb.Th), trabecular number (Tb.N), and trabecular separation (Tb.Sp) as previously describe.[17]

\section{Statistical analysis}

Data are expressed as mean $\pm \mathrm{SD}$ (standard deviation) with annotations in legends. Comparisons between groups were assessed with one-way ANOVA. Statistical analyses were performed using prism software (version 8.0, San Diego, California, USA). Differences were considered significant at $\mathrm{P}<0.05$.

\section{Results}

\section{Experiment 1: preparing simvastatin microcrystals by wet media milling method}

\section{Characterization of microcrystals}

The particle size of simvastatin microcrystals is shown in Table 3. The mean particle size is $1.365 \mu \mathrm{m}$. The $\mathrm{D}_{10}, \mathrm{D}_{50}$, and $\mathrm{D}_{90}$ showed size of the most particle ranged from $0.226-3.425 \mu \mathrm{m}$. The moisture and related substances were controlled well. These data showed we successfully made simvastatin microcrystals. As demonstrated in Fig 1, precipitations can be seen in solutions of $5 \mathrm{mg}$ or $10 \mathrm{mg} / \mathrm{L}$ simvastatin after intense shaking. However, solutions of simvastatin microcrystals look clear even without shaking.

\section{Table 3. Characteristics of simvastatin microcrystals.}

\begin{tabular}{|c|c|c|}
\hline \multicolumn{2}{|c|}{ Characteristics } & Data \\
\hline \multicolumn{2}{|c|}{ Character } & White power \\
\hline \multicolumn{2}{|c|}{$\mathrm{pH}$} & $5.72 \pm 0.05$ \\
\hline \multicolumn{2}{|c|}{ Related substances } & total impurities: $0.433 \%$ \\
\hline \multirow{4}{*}{ Partical size } & $\begin{array}{l}\text { Mean particle } \\
\operatorname{size}(\mu \mathrm{m})\end{array}$ & 1.365 \\
\hline & $\mathrm{D}_{10}(\mu \mathrm{m})$ & 0.226 \\
\hline & $\mathrm{D}_{50}(\mu \mathrm{m})$ & 0.780 \\
\hline & $\mathrm{D}_{90}(\mu \mathrm{m})$ & 3.425 \\
\hline \multicolumn{2}{|c|}{ Moisture } & $2.57 \pm 0.21$ \\
\hline \multicolumn{2}{|c|}{ Content } & Simvastatin $\left(\mathrm{C}_{25} \mathrm{H}_{38} \mathrm{O}_{5}\right): \quad 96.9 \%$ \\
\hline
\end{tabular}




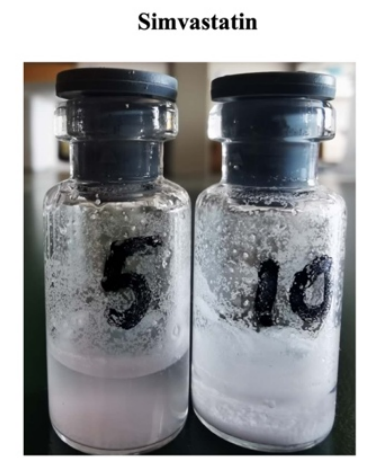

Simvastatin microcrystals

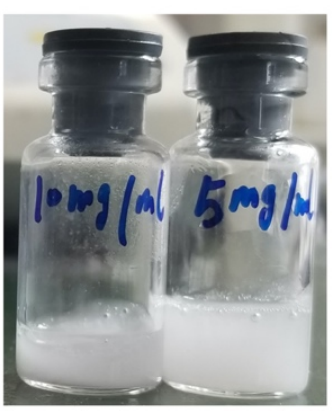

Figure 1. Simvastatin and simvastatin microcrystals $(5 \mathrm{mg} / \mathrm{ml}$ and $10 \mathrm{mg} / \mathrm{ml})$ in water.

\section{Experiment 2: verifying the osteogenesis effect of simvastatin microcrystals in osteoporosis rat model}

\section{Establishment of the rat model of osteoporosis}

Surgery was successful in all animals and recovery was uneventful. The body mass is significantly increased after OVX surgery compared with Sham group (Fig. 2A). Uterus weight at autopsy demonstrated significant decrease in all groups receiving OVX compared with Sham group (Fig. 2B). The increased body mass and decreased uterus weight indicated that we successfully removed ovaries. Furthermore, left tibia (untreated side) BMD and ultimate force was significantly decreased in all OVX groups compared with Sham group (Fig. 2C, D).
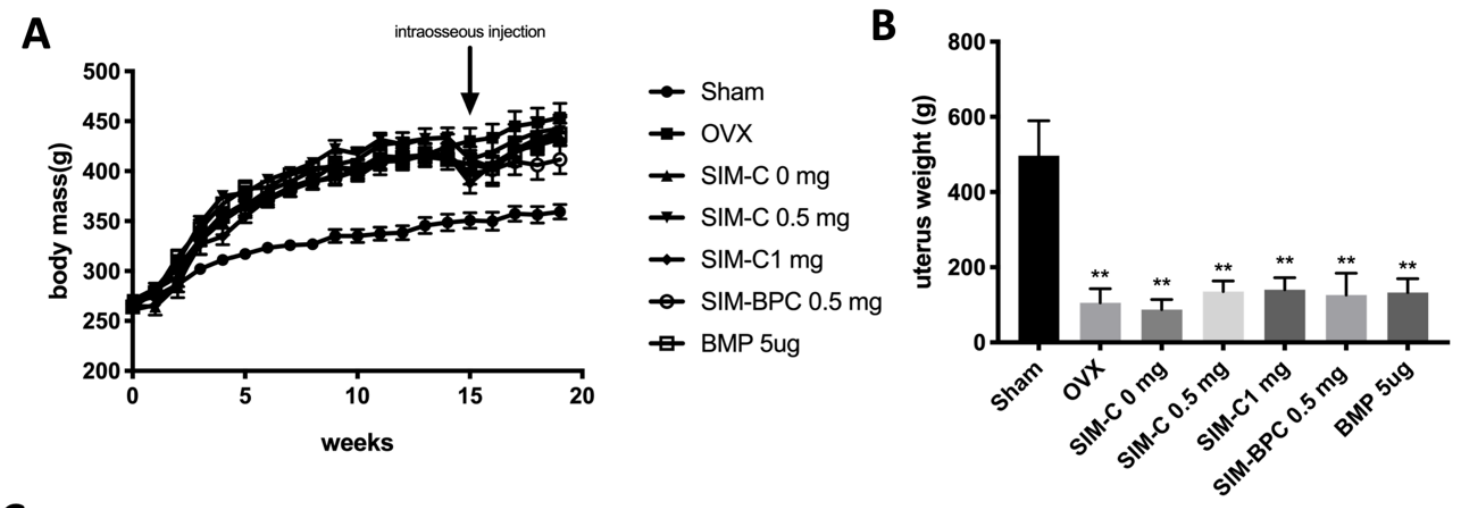

C

D
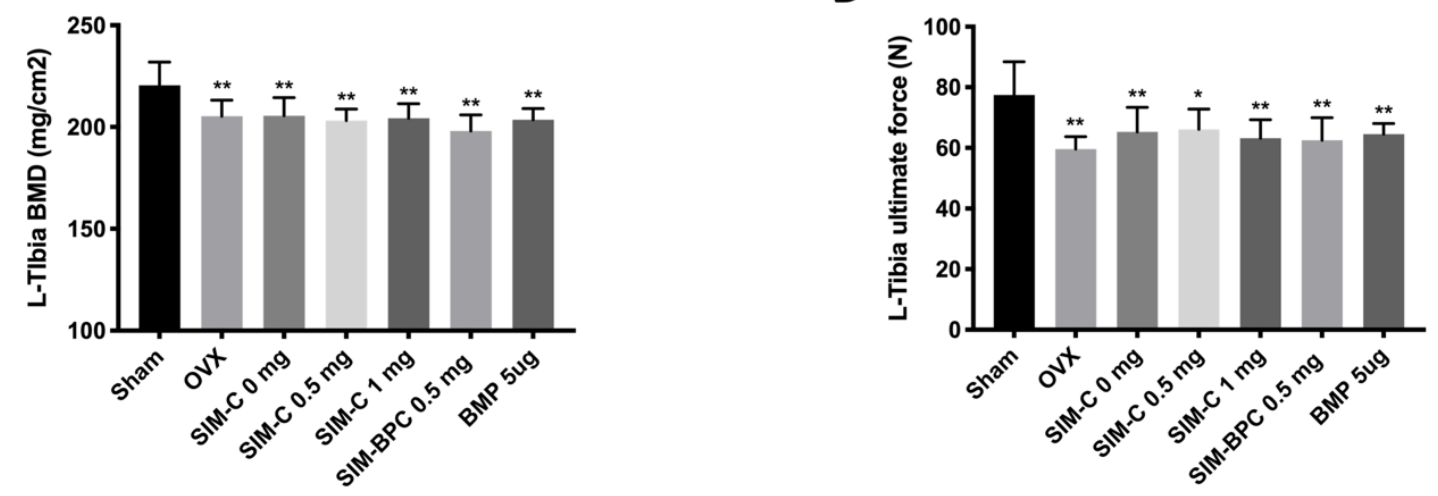
Figure 2. Establishment of the rat model of osteoporosis. (A, B) Body mass and uterus weight of rats after OVX. (C, D) Untreated left tibia BMD and ultimate force results. Statistically significant differences between other groups and Sham group are indicated as follows: $* \mathrm{P}<0.05, * * \mathrm{P}<0.01$. Values represent mean $\pm \mathrm{SD}$. Statistics: one-way ANOVA was used for comparisons.

\section{Right tibia BMD and biomechanical test outcome}

Fig. 3A showed that BMD of right tibia in all drug loaded groups is significantly increased compared with OVX group and SIM-C $0 \mathrm{mg}$ group. Furthermore, ultimate force of tibia in drug loaded groups also improved significantly compared with OVX group and SIM-C $0 \mathrm{mg}$ group (Fig. 3B). And there is no difference in BMD or ultimate force between sham group and drug loaded groups, indicating the BMD of drug loaded groups recovered to a normal level. No differences were observed between BMP and SIM-C groups.

A

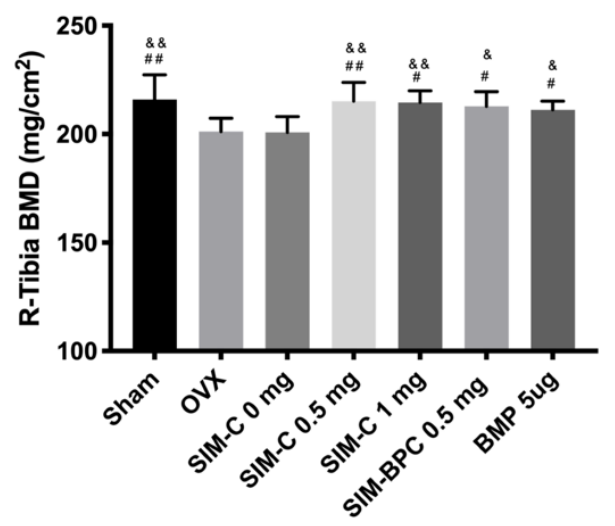

B

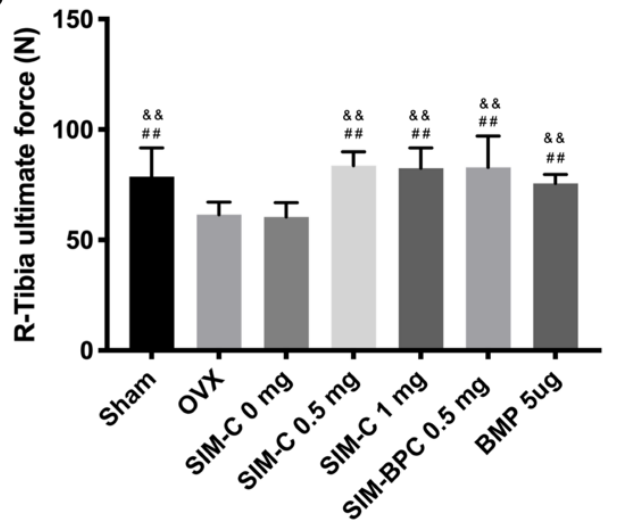

Figure 3. Single intraosseous injection of simvastatin microcrystals improve bone quality. (A) BMD of treated right tibia revealed by DXA. (B) Ultimate force of treated right tibia revealed by three point bending test. Statistically significant differences between other groups and Sham group are indicated as follows: $* \mathrm{P}<0.05, * * \mathrm{P}<0.01$. Significant differences between other groups and the OVX group are indicated as follows: \#P $<0.05$. \#\# $<0.01$. Significant differences between other groups and the SIM-C $0 \mathrm{mg}$ group are indicated as follows: \&P $<0.05$. \&\&P $<$ 0.01. Values represent mean $\pm \mathrm{SD}$. Statistics: one-way ANOVA was used for comparisons.

\section{Experiment 3: verifying the osteogenesis effect of simvastatin microcrystals with CPC in bone defect} rat model

$\mu \mathrm{CT}$

In the SEM images, no obvious difference was observed in the majority of the CPC fracture surfaces between different groups (Fig. 4B). And additional drug power did not alter the solidification time due to our practice. At 8 weeks post operation, we conducted a $\mu \mathrm{CT}$ analysis to determine the defect healing. Fig. 5 shows the typical images of $\mu \mathrm{CT}$, revealing the in vivo implant situation and host bone regeneration in week 8 post-surgery. The CPC group basically kept their original contour. The three-dimensional reconstruction visualized only small signs of material degradation and bone regeneration around implant. 
The contour of CPC implant with loaded drugs was significantly changed and the new bone with thicker and denser trabecula occupied the space of degraded CPC.

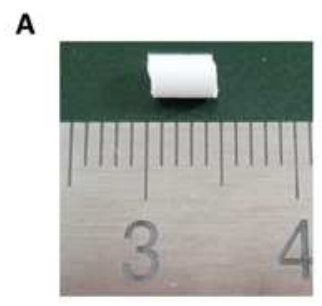

B

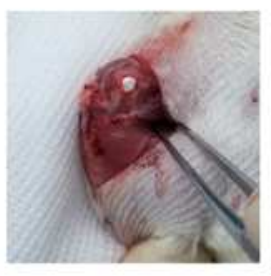

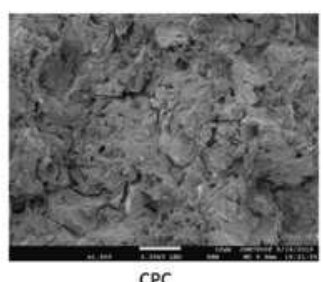

CPC

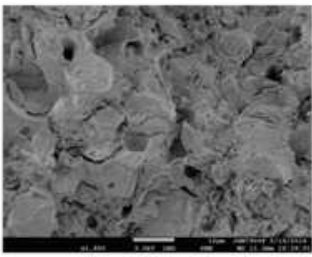

CPC+SIM-C 50 ug

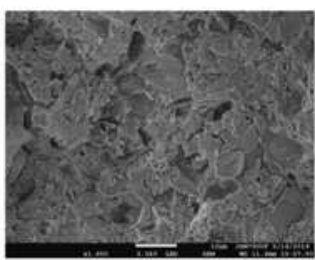

CPC+BMP Sug

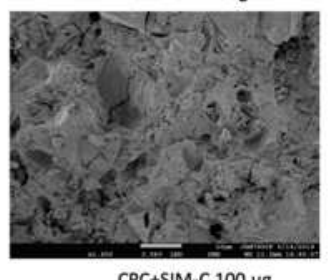

CPC+SIM-C 100 ug

Figure. 4. (A) CPC implant and surgery diagram. (B) SEM image of CPC fracture surface.

The VOI quantitative analysis confirmed that bone regeneration values were significantly improved by the supplement of simvastatin microcrystals or BMP in CPC. BV/TV, Tb.Th, Tb.N were significantly higher in SIM-C 50ug, 100ug and BMP 5ug group compared with CPC group (Fig. 6A-C). Tb.Sp, CV were also decreased in drug group compared with CPC group (Fig. 6D-E).

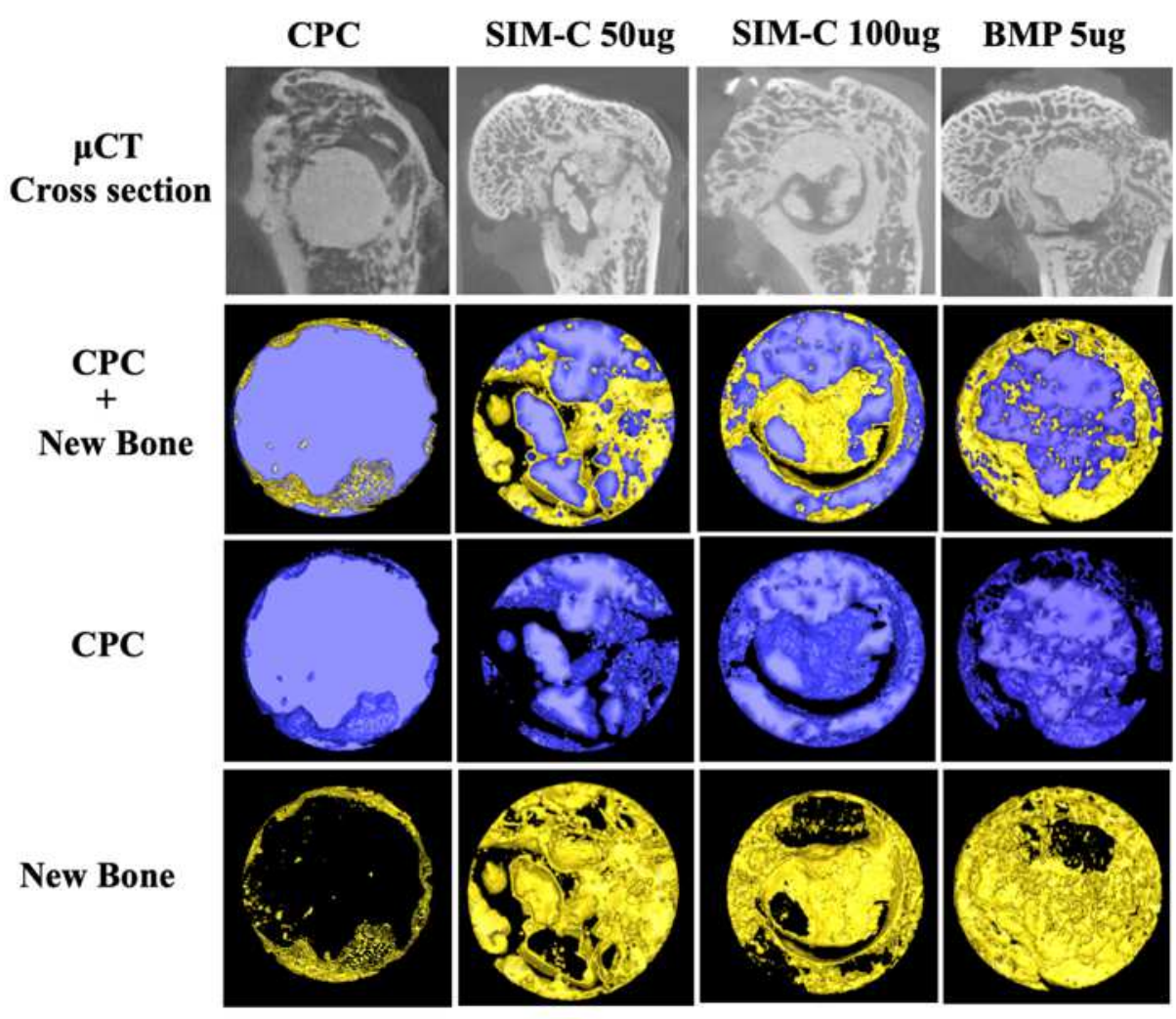


Fig. 5. The $\mu \mathrm{CT}$ images of the upper row are cross sections of implants with their maximum diameter. The other images are three-dimensional reconstruction of VOI showing the implants (blue) and bone (gold).

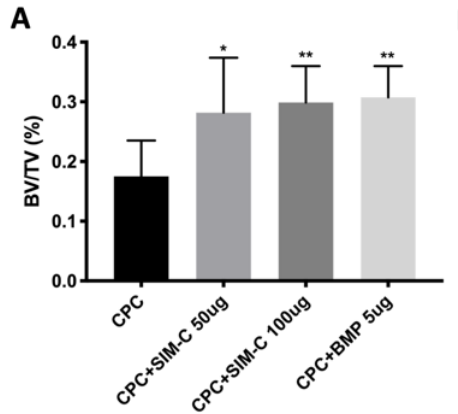

D

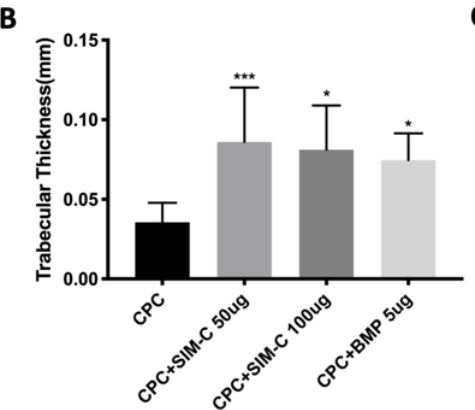

E
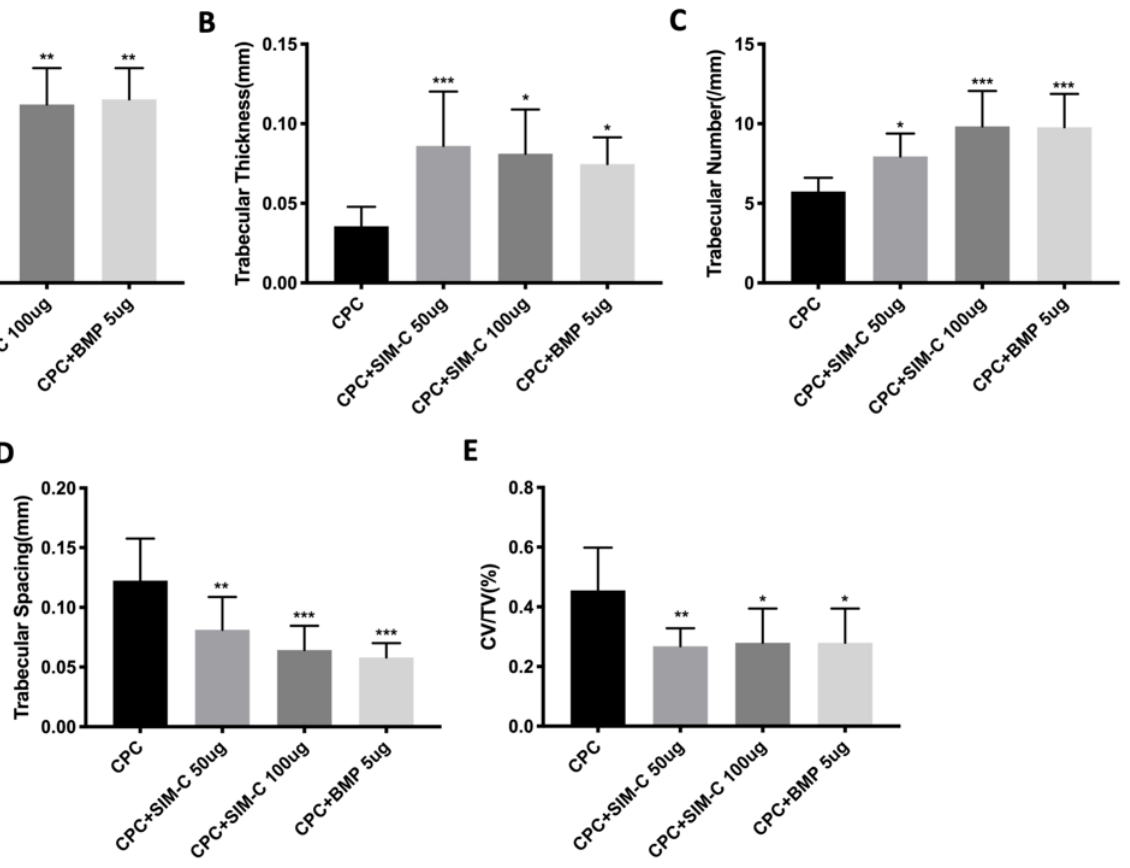

Fig. 6. Quantitative analysis of bone regeneration in VOI of $\mu \mathrm{CT}$ shows values of BV/TV (bone volume/tissue volume), trabecular thickness (Tb.Th), trabecular number (Tb.N), trabecular separation (Tb.Sp), and CV/TV (cement volume/tissue volume). Statistically significant differences between other groups and the vehicle group are indicated as follows: $* \mathrm{P}<0.05, * * \mathrm{P}<0.01$. Values represent mean $\pm \mathrm{SD}$. Statistics: one-way ANOVA was used for comparisons.

\section{Histology}

Bone regeneration of these histological sections was scored on a semiquantitative scale by an individual blinded observer, using a modified scoring method similar to that described previously (Table 4).[18] Representative histological findings of bone formation further proved the results from $\mu \mathrm{CT}$ analysis. Decalcification removed all minerals and CPC implant while kept the tissue. Revealed by HE and Masson staining, abundant newly bone formation and bone matrix with collagen deposition in simvastatin microcrystals and BMP loaded CPC, just a little bone formation in CPC group (Fig. 7). Semiquantitative scale demonstrated a higher bone regeneration score in drug loaded CPC group (Fig. 8).

Table 4 Semiquantitative scale for evaluation of bone regeneration.

\begin{tabular}{cl}
\hline Score & Extent of new bone in defect \\
\hline 0 & No bone formation \\
1 & Minimal bone formation (only very small portion in the defect) \\
2 & Low bone formation (less than one-fourth of the defect) \\
3 & Moderate bone formation
\end{tabular}


(less than one-half and more than one-fourth of the defect)

Abundant bone formation

4

(less than three-quarter and more than one-half of the defect)

5

Extensive bone formation

(more than three-quarter of the defect)

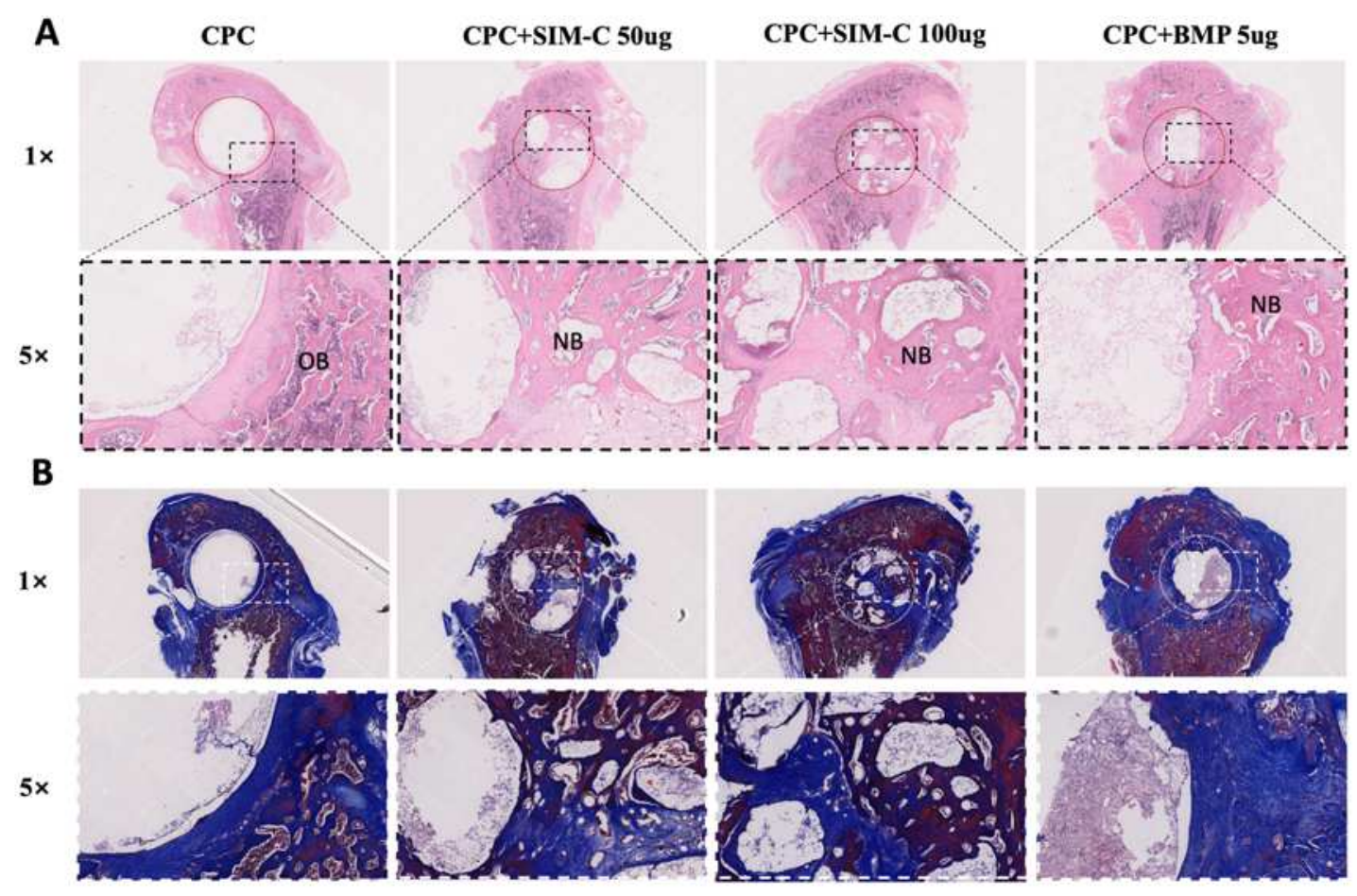

Fig. 7. HE and Masson's trichrome staining shows histological features of the defect area after decalcification. (A) HE staining, (B) Masson's trichrome staining. OB: old bone. NB: new bone.

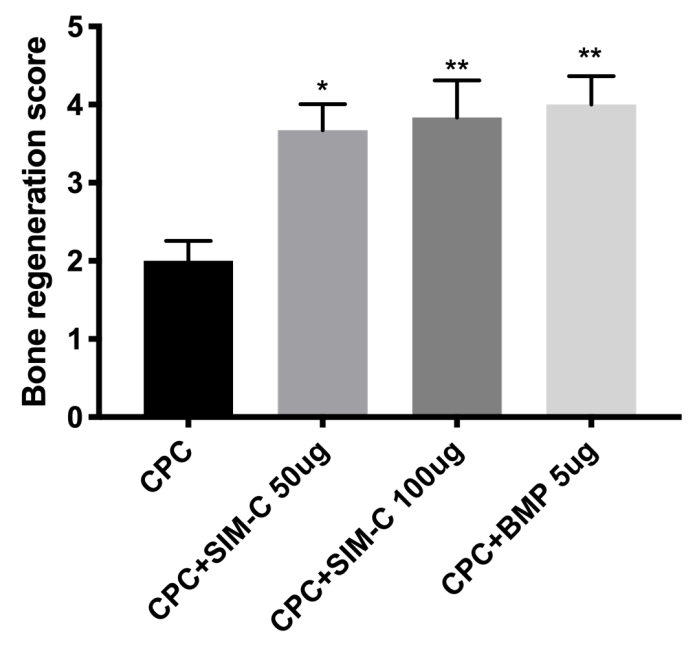


Fig. 8. Bone regeneration score evaluated by a semiquantitative scale. Statistically significant differences between other groups and the CPC group are indicated as follows: $* \mathrm{P}<0.05, * * \mathrm{P}<0.01$. Values represent mean $\pm \mathrm{SD}$. Statistics: oneway ANOVA was used for comparisons.

\section{Serum assay}

There is no significant difference in serum P1NP and TRACP5b between groups at the same time point (Fig. 9A, B)

A

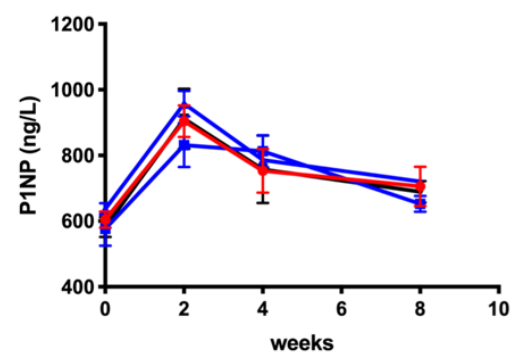

B

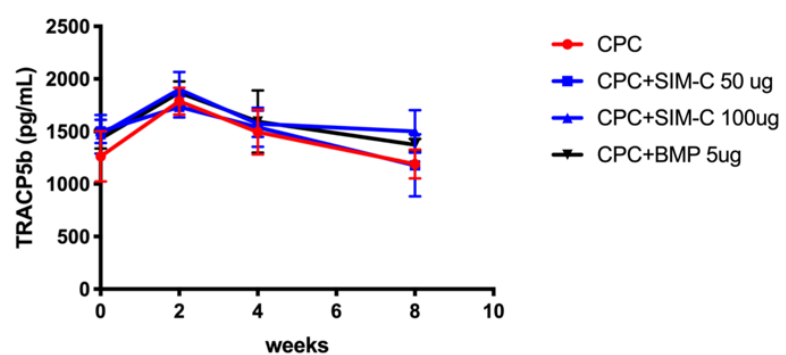

Fig. 9. Serum bone formation marker, intact N-terminal propeptide of type-1 procollagen (P1NP) and bone resorption marker, tartrate-resistant acid phosphatase 5b (TRACP 5b) in different groups. All data are expressed as mean \pm SD.

\section{Discussion}

Approaches to regenerate bone include specific grafting surgical techniques with autogenous bone, substitutes, barrier membranes, growth factors, stem cell therapy, and lately the osteopromotive pharmacological compounds. The pharmacological approaches have gained popularity owing to their convenience and the advantageous cost-effectiveness when applied with other grafting techniques.[19] A number of studies have demonstrated that simvastatin can significantly increase bone formation. [7, 15] In addition to direct effect on osteoblasts and osteoclast, the underlying mechanisms are complexed by statins' pleiotropic effects including anti-inflammation, angiogenesis and antibacterial activity which could also play an important role in bone formation. $[3,20]$ Despite of the unclear mechanism, abundant in vivo studies have shown the positive effect of simvastatin on bone formation. Locally application of simvastatin can improve osteoporosis in ovariectomized rats[6] and minipigs.[8] Also, local delivery of simvastatin through controlled drug delivery systems with much lower doses and dosages are effective in fracture healing.[20] Both a $10 \mathrm{mg} / \mathrm{kg}$ intraperitoneal injection of simvastatin daily for 30 days[21] and an oral dosage of 5 $\mathrm{mg} / \mathrm{kg} / \mathrm{d}$ for 28 days[22] improved osseointegration in osteoporotic rats.

As simvastatin has been used as lipid-lowering medicine safely for many years. It seems very promising that statins could be translated into clinic. However, after decades of studying, we are still stay in preclinical phases. Only in the scope of dentistry, clinical studies have investigated the potential of simvastatin 
in bone, soft tissue, and cartilage regeneration or protection. For instance, Ranjan et al. demonstrated 1.2 mg simvastatin gel can improve osseous defect healing in chronic periodontitis.[23] For this drug, route of administration really matters. As mentioned in introduction, after the first-pass hepatic metabolism, only approximately $5 \%$ of the active compound remains in the circulation, even less at the defect site. However, it is necessary to maintain the effective concentrations of simvastatin in the circulating blood for a long time to be sure that simvastatin is able to continuously affect the bone. Therefore, in the systemic approach, simvastatin needs to be continuously administered for a long period of time, which increases the total dose.[20] A high daily dose is likely elicits unwanted side effects, such as increasing the risk of liver failure, kidney disease, rhabdomyolysis, and myalgia.[3] Local delivery of simvastatin is another option that can be used. Carries that have been reported to deliver simvastatin includes PLGA (poly Lactic-coglycolic acid), collagen, gelatin, TCP (three calcium phosphate), hydrogen. However, these studies, including our previous studies, usually used raw chemicals or oral formulations while additional formulation process is needed for locally application. In this study, we have successfully create simvastatin microcrystals with a mean particle size of $1.365 \mu \mathrm{m}$. And microcrystals significantly increase water solubility of simvastatin witch allow us to dissolve microcrystals easily even in concentration of $10 \mathrm{mg} / \mathrm{ml}$. What's more, microcrystals can be easily industrialized as a mature pharmaceutical preparation process. However, due to the unclear mechanism how simvastatin promotes bone formation, it's necessary to verify whether simvastatin microcrystals remain the osteogenic effect.

We showed single intraosseous injection of simvastatin microcrystals with $0.5 \mathrm{mg}$ or $1 \mathrm{mg}$ could improve bone quality of tibia as well as BMP 5 ug. Both BMD and strength were increased in treated tibia. However, we did not observe any difference in BMD and strength between SIM-C $0.5 \mathrm{mg}$ and SIM-BPC $0.5 \mathrm{mg}$ group although the water solubility is increased in microcrystal form. Despite all this, we proved simvastatin remains the ability to promote bone formation after making it into microcrystals. In bone defect experiment, 50 ug and 100 ug simvastatin microcrystals also promoted defect healing compared with the vehicle group. And BMP 5 ug group did not show better healing than simvastatin microcrystal group. Thus, this study showed formulation of simvastatin microcrystals could be effective with poloxamer or CPC which are both potential to be approved by China Food and Drug Administration.

There are some limitations to the present study. First, we did not study simvastatin microcrystals release in vitro and retention in vivo, although we previously showed no initial burst release was observed during the first $24 \mathrm{~h}$ in simvastatin/poloxamer 407 hydrogels. [8] To further translation, a precise pharmacodynamic study for microcrystals is needed. Second, the optimal dose for local usage is still not clear due to the limited groups. It seems the dose of simvastatin could be still decreased to some extent. Our big animal study showed 0.5 and $1 \mathrm{mg}$ injection of simvastatin in minipig vertebrae is effective to enhance implant fixation and improve bone quality.[8,9] Our unpublished data showed 3D-printed porous cages with 0.5 $\mathrm{mg}$ simvastatin in hydrogel can promote bone ingrowth and spinal fusion in adult male rhesus macaques. The possible overdose in this study may also explain the phenomenon that we did not find any difference between SIM-C $0.5 \mathrm{mg}$ and SIM-BPC $0.5 \mathrm{mg}$ group in osteoporosis model.

\section{Conclusion}

We successfully prepared simvastatin microcrystals with a mean particle size of $1.365 \mu \mathrm{m}$ by wet media 
milling method. And simvastatin microcrystals remain the osteogenic ability both in osteoporosis model and bone defect model. It might provide an adjunctive method to augment local osteoporotic bone, reduce the incidence of osteoporotic fractures, or improve bone defect healing.

\begin{abstract}
Abbreviations
OVX: ovariectomy; BMD: bone mineral density; CPC: calcium phosphate cements; BMP: bone morphogenetic protein; BV/TV: bone volume/tissue volume; Tb.Th: trabecular thickness; Tb.N: trabecular number; Tb.Sp: trabecular separation; CV/TV: cement volume/tissue volume; HPLC: high-performance liquid chromatography: BPC: bulk pharmaceutical chemicals; PBS: phosphate-buffered saline; DXA: dualenergy X-ray absorptiometry; P1NP: intact N-terminal propeptide of type-1 procollagen; TRACP $5 b$ : tartrate-resistant acid phosphatase 5b; SD: standard deviation; PLGA: poly Lactic-co-glycolic acid: TCP: three calcium phosphate.
\end{abstract}

\title{
Declarations
}

Ethics approval and consent to participate

All experimental procedures and protocols were approved by Peking University Third Hospital Committee on Ethics in the Care and Use of Laboratory Animals (LA2016304).

\section{Consent for publication}

Not applicable.

\section{Availability of data and materials}

All the data generated or analyzed during this study are included in this published article.

\section{Competing interests}

The authors declare that they have no competing interests.

\section{Funding}

This work was supported by grants from the National Natural Science Foundation of China (Project No. 81874010) and National High-tech R\&D Program (Project No. 2015AA020304).

\section{Authors' contributions}

Z.J. participated in the establishment of the animal model and drafted the manuscript. Z.C., J.J. and W.H. were engaged in methodology and animal management. W.C. helped with preparation of simvastatin microcrystals. X.Y., L.H., Z.Q., S.C. were also involved in the writing process and reviewed the final version.

\section{Acknowledgements}

We are grateful for the technical support provided by Peking University Third Hospital Central Laboratory. 
Author details

${ }^{1}$ Department of Orthopedics, Peking University Third Hospital, Beijing 100191, China; ${ }^{2}$ Beijing Key Laboratory of Spinal Diseases, Beijing 100191, China; ${ }^{3}$ Department of Neurology, Peking University Third Hospital, Beijing 100191, China; ${ }^{4}$ Beijing Delivery Pharm Technology Co., Ltd, Beijing 102101, China; ${ }^{5}$ Department of Pharmaceutics, School of Pharmaceutical Sciences, Peking University, Beijing 100191, China.

\section{References}

1. Dimitriou R, Mataliotakis GI, Angoules AG, Kanakaris NK, Giannoudis PV: Complications following autologous bone graft harvesting from the iliac crest and using the RIA: a systematic review. Injury 2011, 42 Suppl 2:S3-15.

2. Mundy G, Garrett R, Harris S, Chan J, Chen D, Rossini G, Boyce B, Zhao M, Gutierrez G: Stimulation of bone formation in vitro and in rodents by statins. Science 1999, 286:1946-1949.

3. Shah SR, Werlang CA, Kasper FK, Mikos AG: Novel applications of statins for bone regeneration. Natl Sci Rev 2015, 2:85-99.

4. Yao W, Farmer R, Cooper R, Chmielewski PA, Tian XY, Setterberg RB, Jee WS, Lundy MW: Simvastatin did not prevent nor restore ovariectomy-induced bone loss in adult rats. $J$ Musculoskelet Neuronal Interact 2006, 6:277-283.

5. Obi C, Wysokinski W, Karnicki K, Owen WG, McBane RD, 2nd: Inhibition of platelet-rich arterial thrombus in vivo: acute antithrombotic effect of intravenous HMG-CoA reductase therapy. Arterioscler Thromb Vasc Bio/2009, 29:1271-1276.

6. Yang N, Cui Y, Tan J, Fu X, Han X, Leng H, Song C: Local injection of a single dose of simvastatin augments osteoporotic bone mass in ovariectomized rats. J Bone Miner Metab 2014, 32:252-260.

7. Yueyi C, Xiaoguang H, Jingying W, Quansheng S, Jie T, Xin F, Yingsheng X, Chunli S: Calvarial defect healing by recruitment of autogenous osteogenic stem cells using locally applied simvastatin. Biomaterials 2013, 34:9373-9380.

8. Tan J, Fu X, Sun CG, Liu C, Zhang XH, Cui YY, Guo Q, Ma T, Wang H, Du GH, et al: A single CT-guided percutaneous intraosseous injection of thermosensitive simvastatin/poloxamer 407 hydrogel enhances vertebral bone formation in ovariectomized minipigs. Osteoporos Int 2016, 27:757-767.

9. Fu X, Tan J, Sun CG, Leng HJ, Xu YS, Song CL: Intraosseous Injection of Simvastatin in Poloxamer 407 Hydrogel Improves Pedicle-Screw Fixation in Ovariectomized Minipigs. J Bone Joint Surg Am 2016, 98:1924-1932.

10. Othman R, Vladisavljević GT, Simone E, Nagy ZK, Holdich RG: Preparation of Microcrystals of Piroxicam Monohydrate by Antisolvent Precipitation via Microfabricated Metallic Membranes with Ordered Pore Arrays. Cryst Growth Des 2017, 17:6692-6702.

11. Sun R, Guo Y, Yin N, Yin J, Yin T, He H, Gou J, Zhang Y, Tang X: Preparation of sterile longacting injectable medroxyprogesterone acetate microcrystals based on anti-solvent 
precipitation and crystal habit control. Expert Opin Drug Deliv 2019, 16:1133-1144.

12. Pillai $O$, Panchagnula R: Transdermal delivery of insulin from poloxamer gel: ex vivo and in vivo skin permeation studies in rat using iontophoresis and chemical enhancers. $J$ Control Release 2003, 89:127-140.

13. Djekic L, Krajisnik D, Martinovic M, Djordjevic D, Primorac M: Characterization of gelation process and drug release profile of thermosensitive liquid lecithin/poloxamer 407 based gels as carriers for percutaneous delivery of ibuprofen. Int J Pharm 2015, 490:180-189.

14. Giuliano E, Paolino D, Fresta M, Cosco D: Mucosal Applications of Poloxamer 407-Based Hydrogels: An Overview. Pharmaceutics 2018, 10.

15. Li X, Song QS, Wang JY, Leng HJ, Chen ZQ, Liu ZJ, Dang GT, Song CL: Simvastatin induces estrogen receptor-alpha expression in bone, restores bone loss, and decreases ERalpha expression and uterine wet weight in ovariectomized rats. J Bone Miner Metab 2011, 29:396-403.

16. Zhang W, Liu C, Hai B, Du G, Wang H, Leng H, Xu Y, Song C: A Convenient In Vivo Model Using Small Interfering RNA Silencing to Rapidly Assess Skeletal Gene Function. PLOS One 2016, 11:e0167222.

17. Kuang GM, Yau WP, Wu J, Yeung KW, Pan H, Lam WM, Lu WW, Chiu KY: Strontium exerts dual effects on calcium phosphate cement: Accelerating the degradation and enhancing the osteoconductivity both in vitro and in vivo. J Biomed Mater Res A 2015, 103:1613-1621.

18. Zhang $Y$, Cheng N, Miron R, Shi B, Cheng X: Delivery of PDGF-B and BMP-7 by mesoporous bioglass/silk fibrin scaffolds for the repair of osteoporotic defects. Biomaterials 2012, 33:6698-6708.

19. Gupta S, Del Fabbro M, Chang J: The impact of simvastatin intervention on the healing of bone, soft tissue, and TMJ cartilage in dentistry: a systematic review and metaanalysis. Int J Implant Dent 2019, 5:17.

20. Moshiri A, Sharifi AM, Oryan A: Role of Simvastatin on fracture healing and osteoporosis: a systematic review on in vivo investigations. Clin Exp Pharmacol Physio/ 2016, 43:659684.

21. Ayukawa $\mathrm{Y}$, Okamura A, Koyano K: Simvastatin promotes osteogenesis around titanium implants. Clin Oral Implants Res 2004, 15:346-350.

22. Du Z, Chen J, Yan F, Xiao Y: Effects of Simvastatin on bone healing around titanium implants in osteoporotic rats. Clin Oral Implants Res 2009, 20:145-150.

23. Ranjan R, Patil SR, H RV: Effect of in-situ application of simvastatin gel in surgical management of osseous defects in chronic periodontitis-A randomized clinical trial. $J$ Oral Biol Craniofac Res 2017, 7:113-118. 
Figures

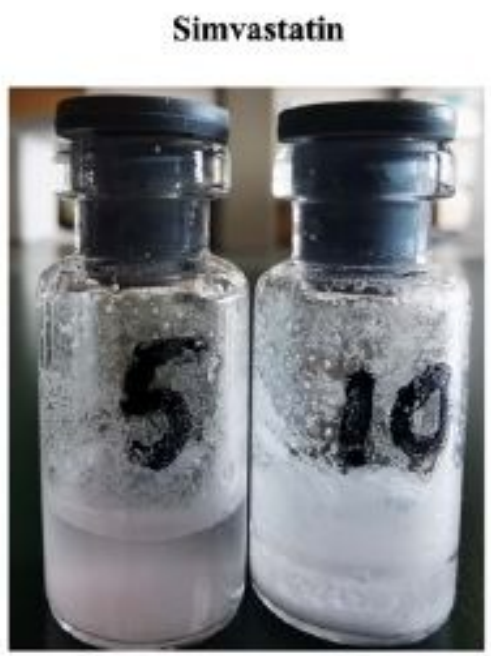

Simvastatin microcrystals

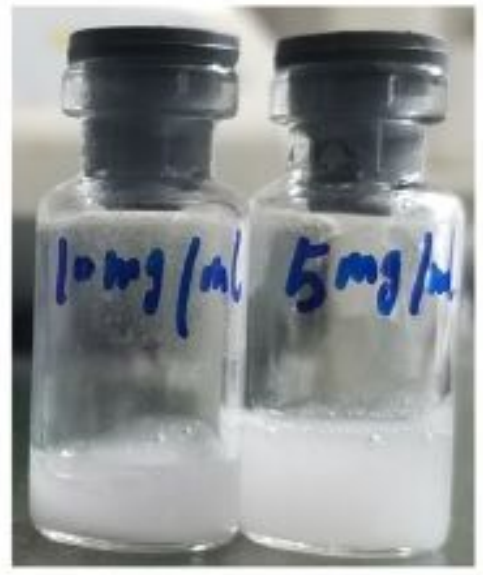

Figure 1

Simvastatin and simvastatin microcrystals $(5 \mathrm{mg} / \mathrm{ml}$ and $10 \mathrm{mg} / \mathrm{ml})$ in water. 
A

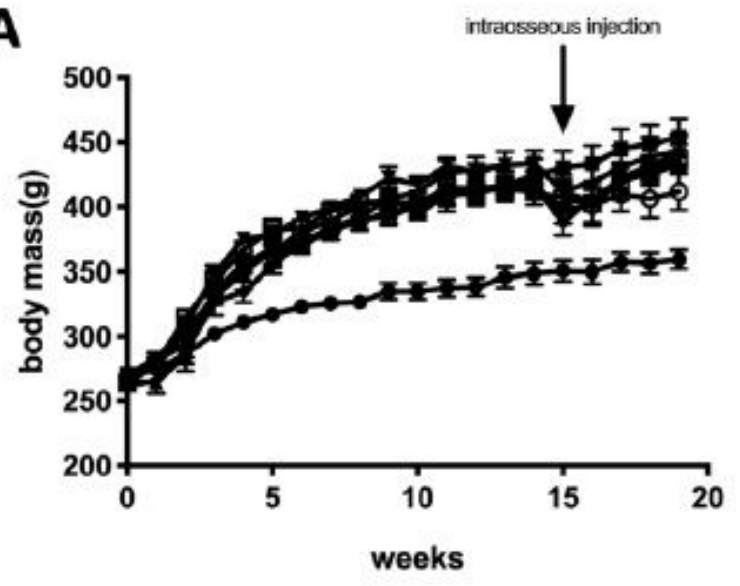

C

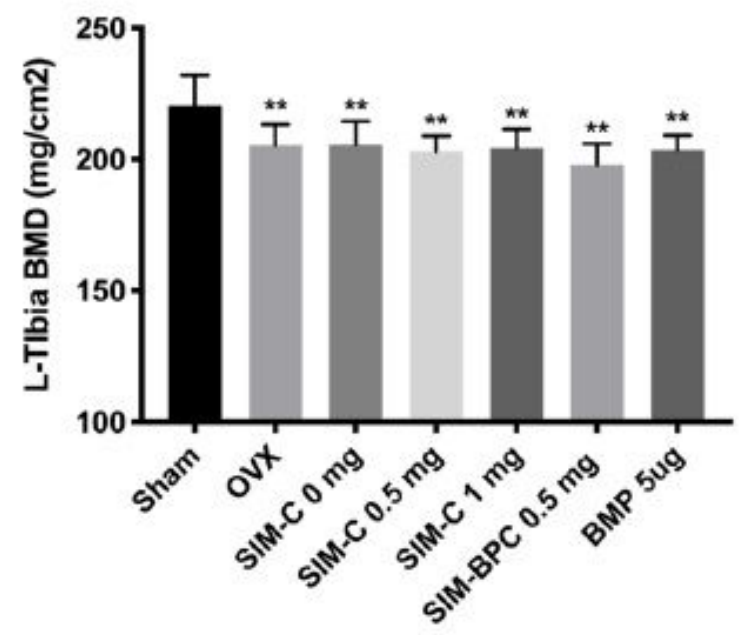

B

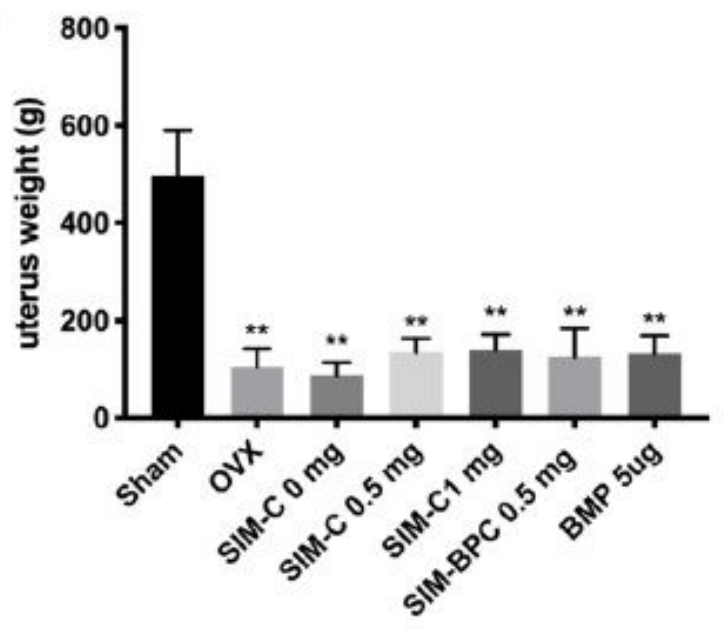

D

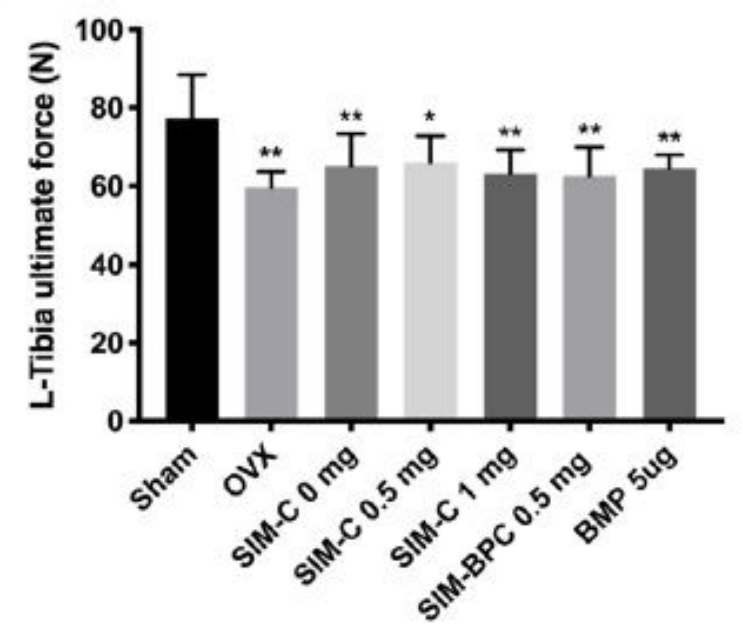

Figure 2

Establishment of the rat model of osteoporosis. (A, B) Body mass and uterus weight of rats after OVX. (C, D) Untreated left tibia BMD and ultimate force results. Statistically significant differences between other groups and Sham group are indicated as follows: ${ }^{*} P<0.05,{ }^{\star \star} P<0.01$. Values represent mean $\pm S D$. Statistics: one-way ANOVA was used for comparisons. 
A

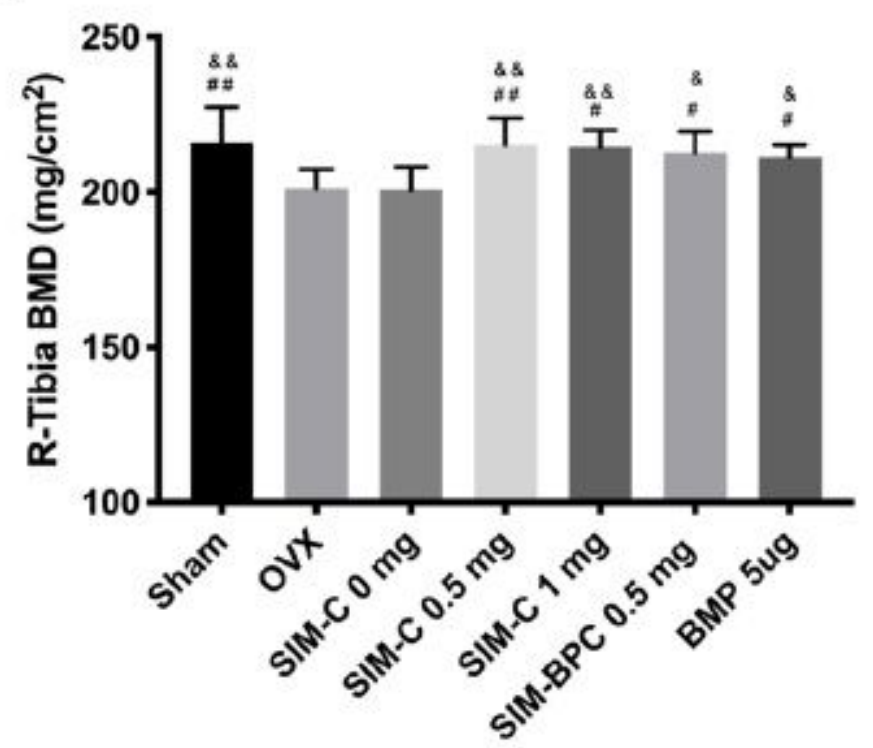

B

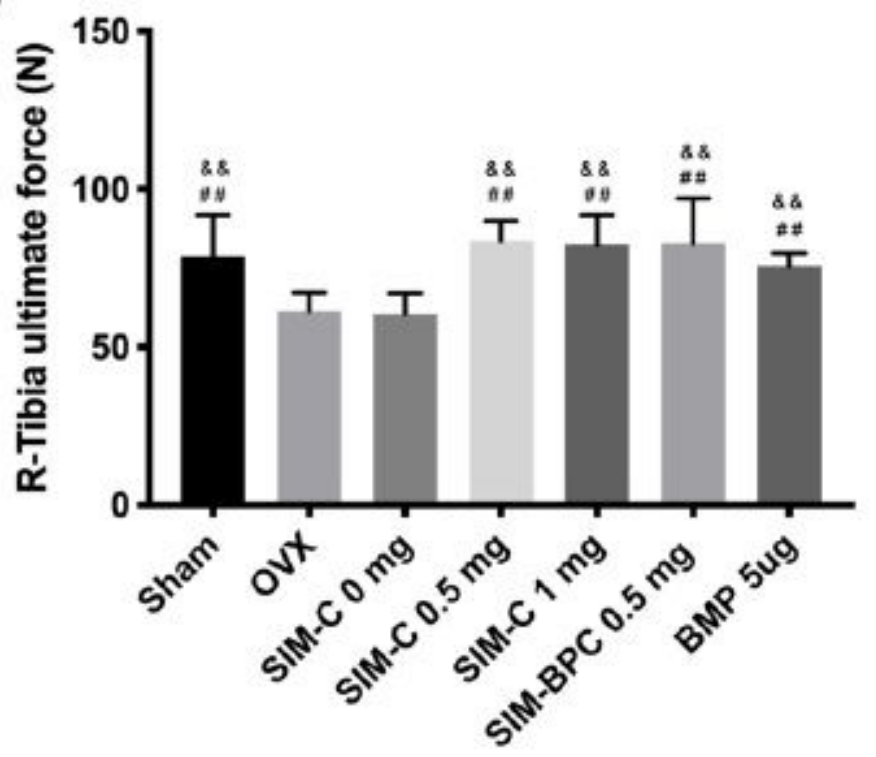

Figure 3

Single intraosseous injection of simvastatin microcrystals improve bone quality. (A) BMD of treated right tibia revealed by DXA. (B) Ultimate force of treated right tibia revealed by three point bending test.

Statistically significant differences between other groups and Sham group are indicated as follows: ${ }^{*} \mathrm{P}$ 0.05 , $* * P<0.01$. Significant differences between other groups and the OVX group are indicated as follows: \#P<0.05. \#\#P<0.01. Significant differences between other groups and the SIM-C $0 \mathrm{mg}$ group are indicated as follows: \&P<0.05. \&\&P<0.01. Values represent mean \pm SD. Statistics: one-way ANOVA was used for comparisons.

A
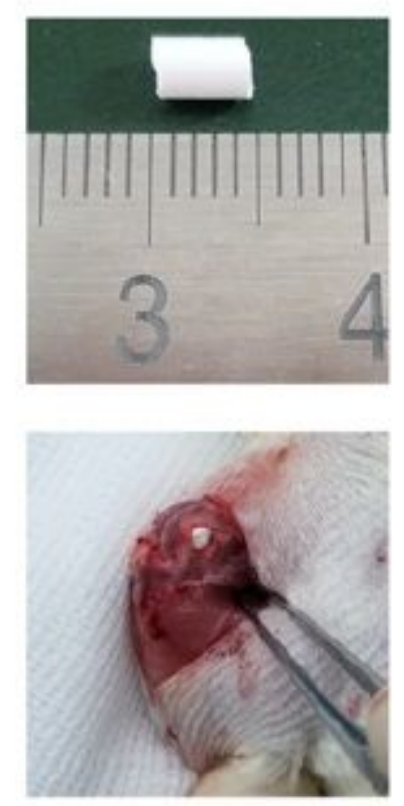

B

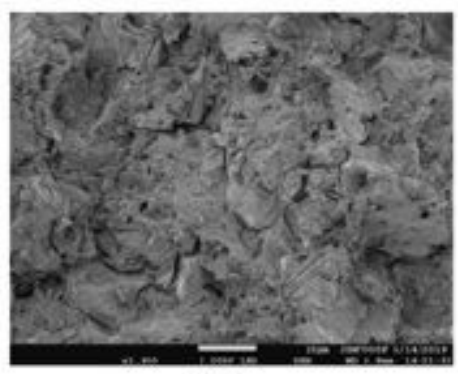

CPC

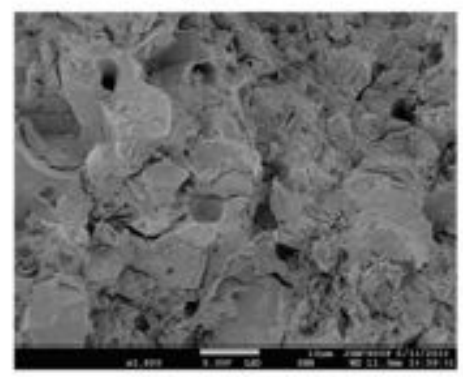

$\mathrm{CPC}+\mathrm{SIM}-\mathrm{C} 50$ ug

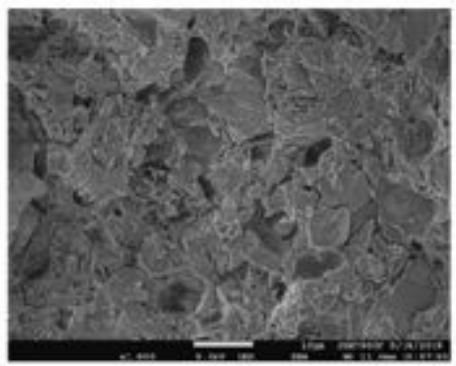

CPC+BMP 5ug

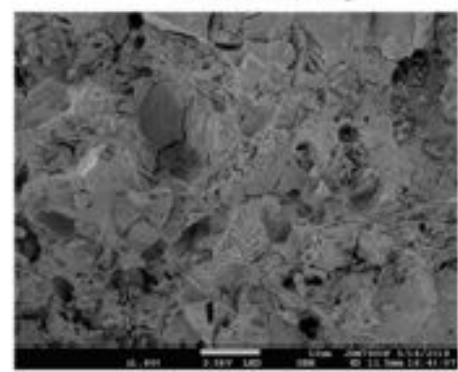

CPC+SIM-C 100 ug

Figure 4 
(A) CPC implant and surgery diagram. (B) SEM image of CPC fracture surface.

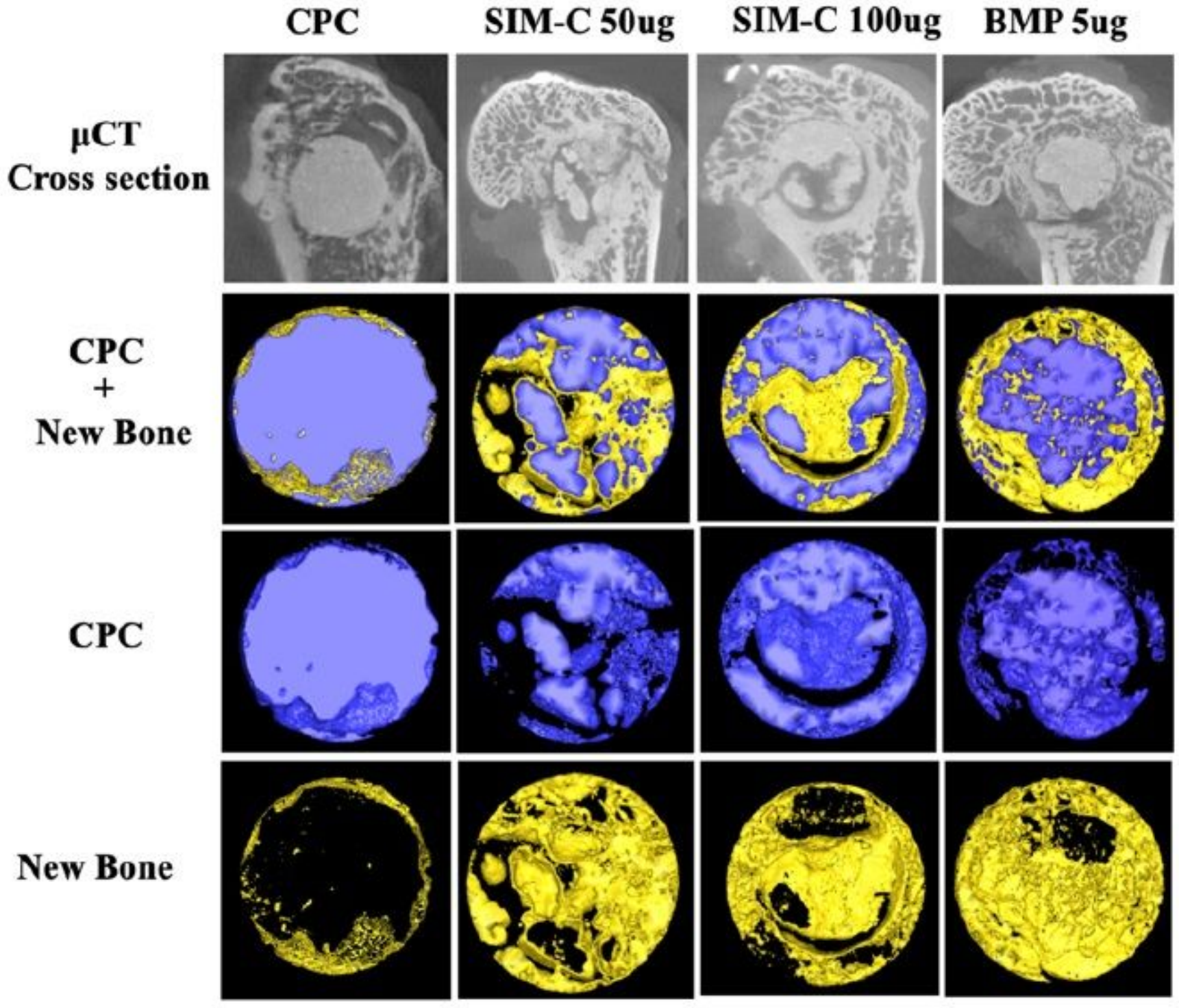

Figure 5

The $\mu \mathrm{CT}$ images of the upper row are cross sections of implants with their maximum diameter. The other images are three-dimensional reconstruction of VOI showing the implants (blue) and bone (gold). 


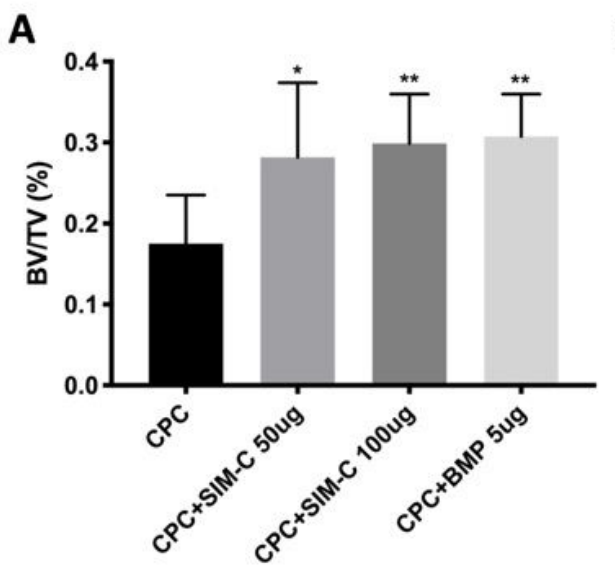

D
B

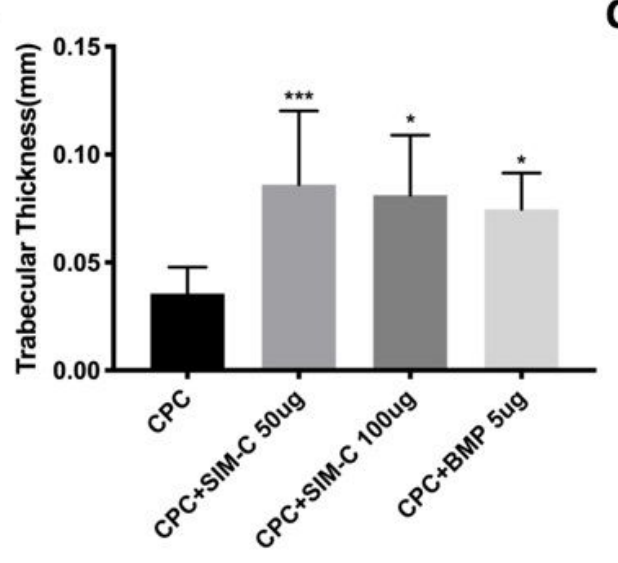

C

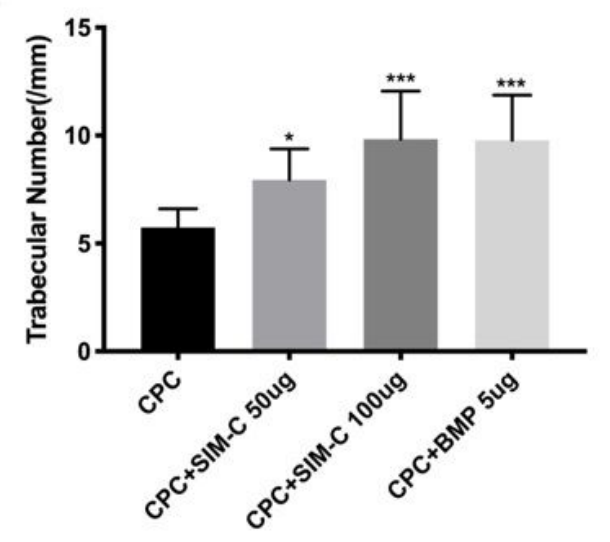

E
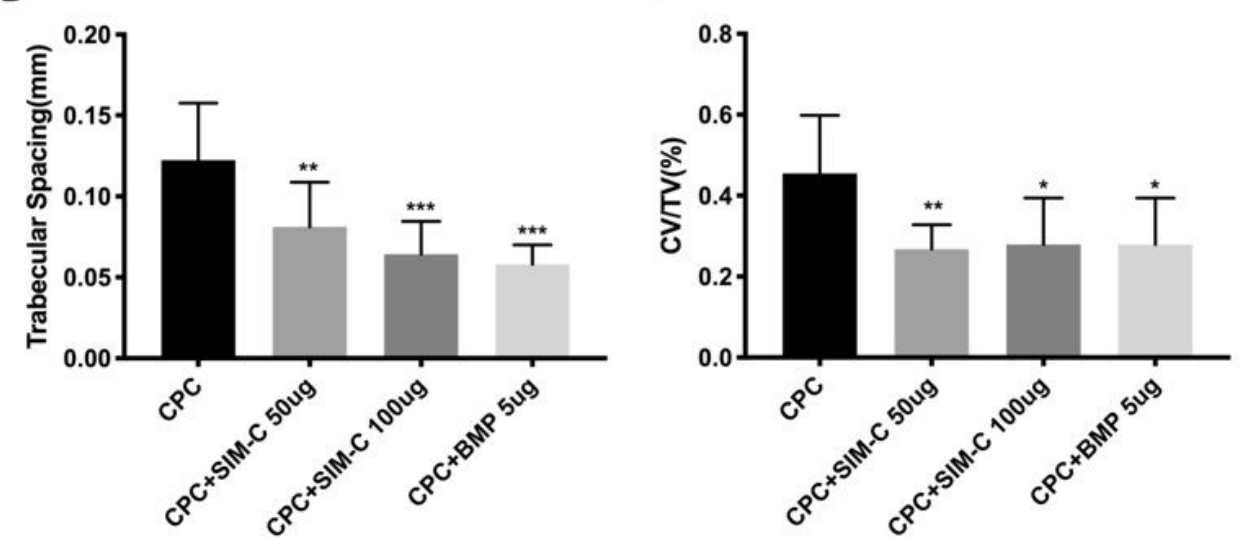

Figure 6

Quantitative analysis of bone regeneration in VOI of $\mu \mathrm{CT}$ shows values of BV/TV (bone volume/tissue volume), trabecular thickness (Tb.Th), trabecular number (Tb.N), trabecular separation (Tb.Sp), and $\mathrm{CV} / \mathrm{TV}$ (cement volume/tissue volume). Statistically significant differences between other groups and the vehicle group are indicated as follows: ${ }^{*} P<0.05$, ${ }^{\star \star} P<0.01$. Values represent mean \pm SD. Statistics: oneway ANOVA was used for comparisons. 
A

CPC

CPC+SIM-C 50ug

CPC+SIM-C 100ug

CPC+BMP 5ug

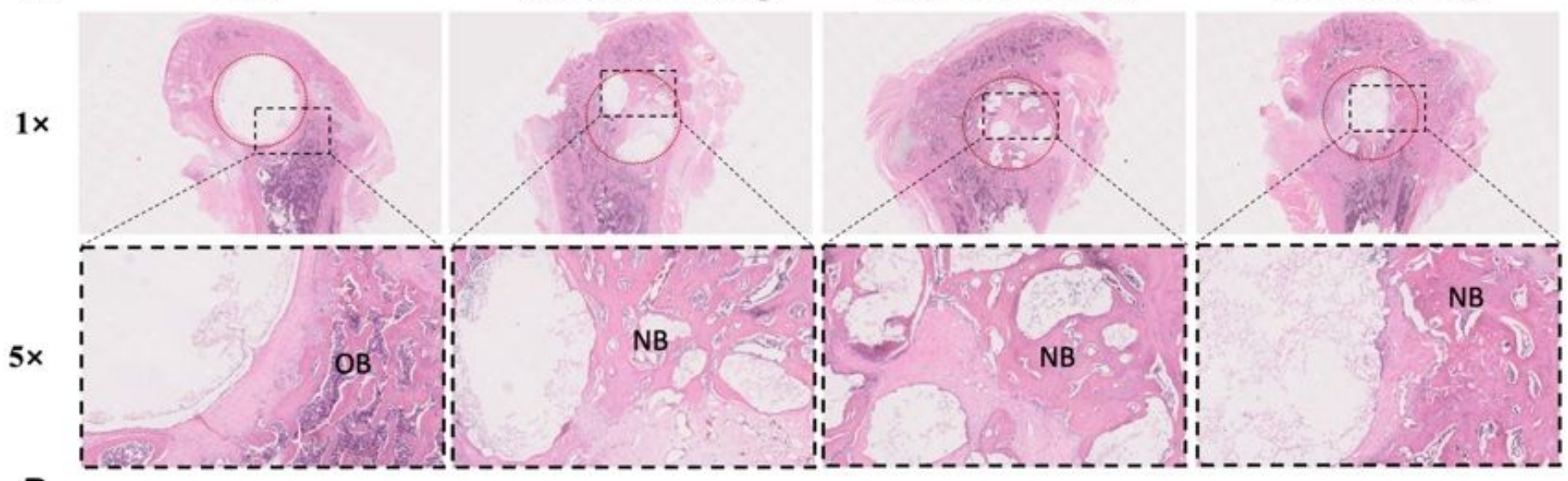

B
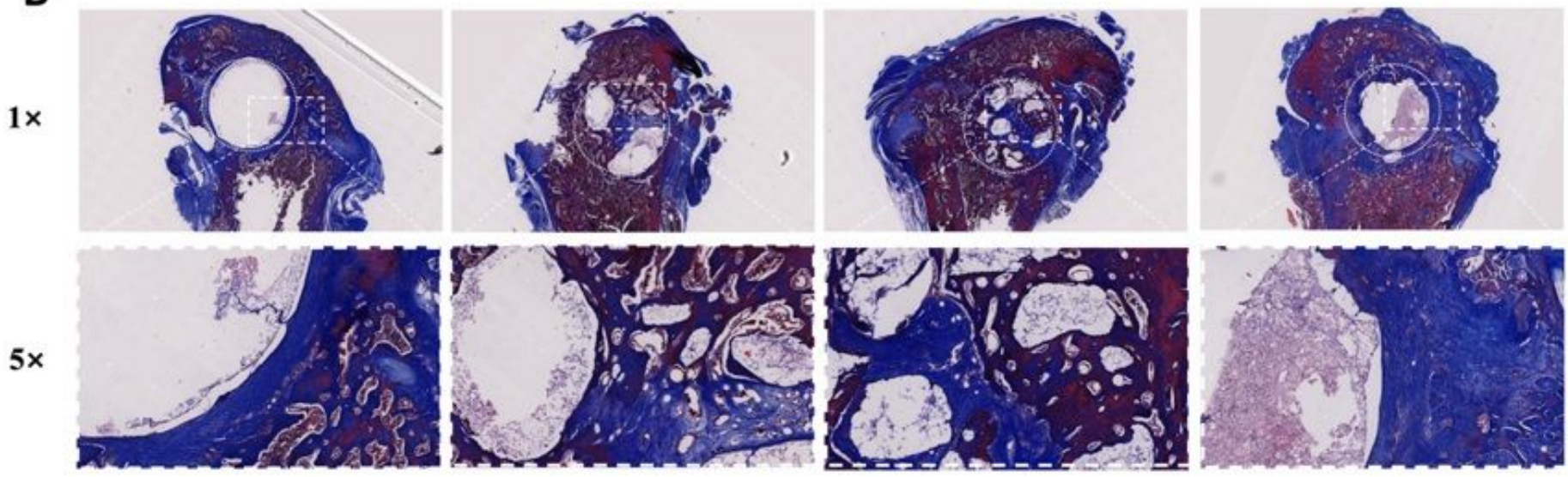

Figure 7

HE and Masson's trichrome staining shows histological features of the defect area after decalcification. (A) HE staining, (B) Masson's trichrome staining. OB: old bone. NB: new bone. 


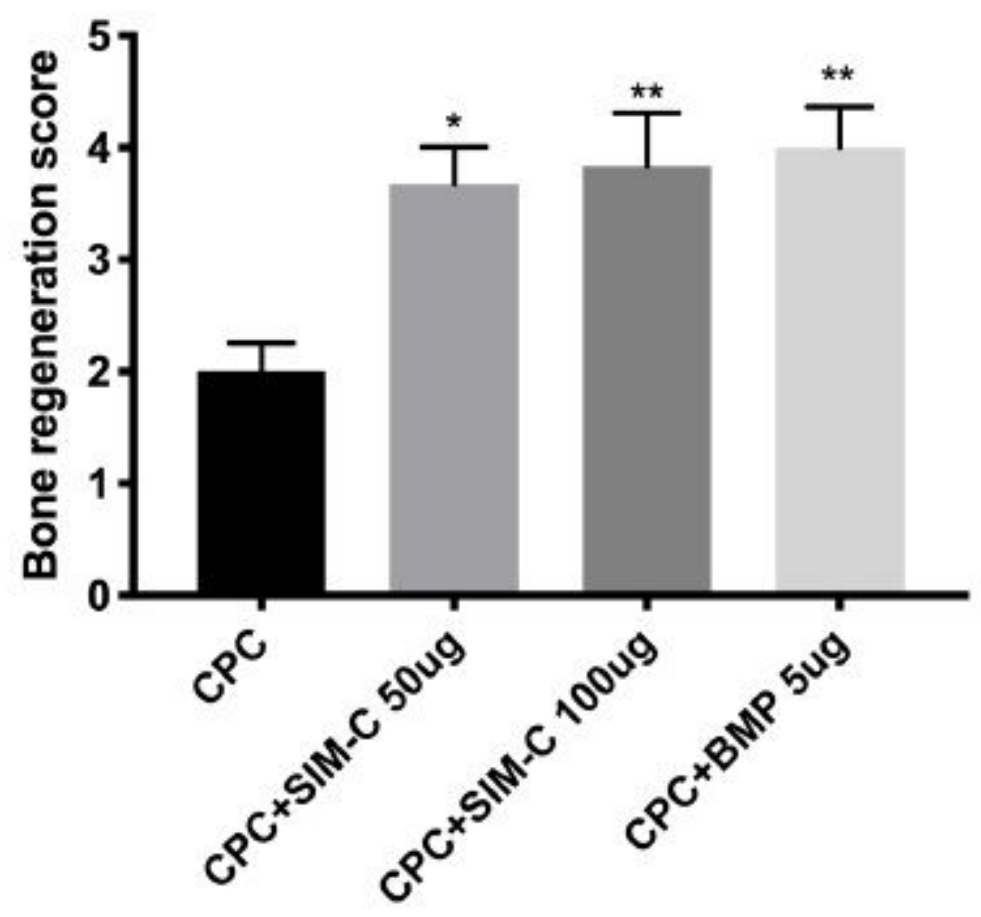

Figure 8

Bone regeneration score evaluated by a semiquantitative scale. Statistically significant differences between other groups and the CPC group are indicated as follows: ${ }^{*} \mathrm{P}<0.05,{ }^{*} \mathrm{P}<0.01$. Values represent mean \pm SD. Statistics: oneway ANOVA was used for comparisons.

A

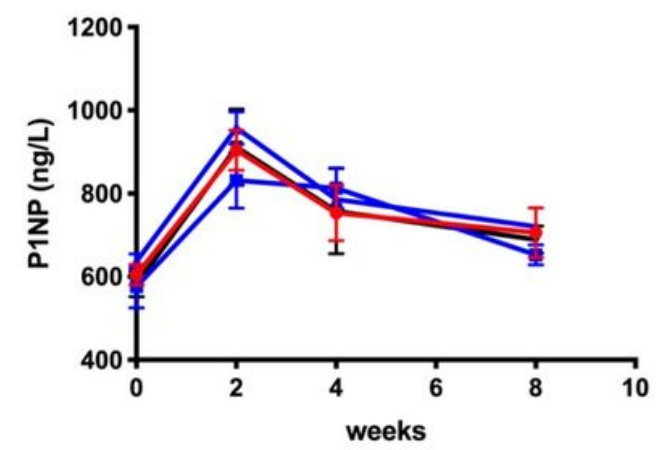

B

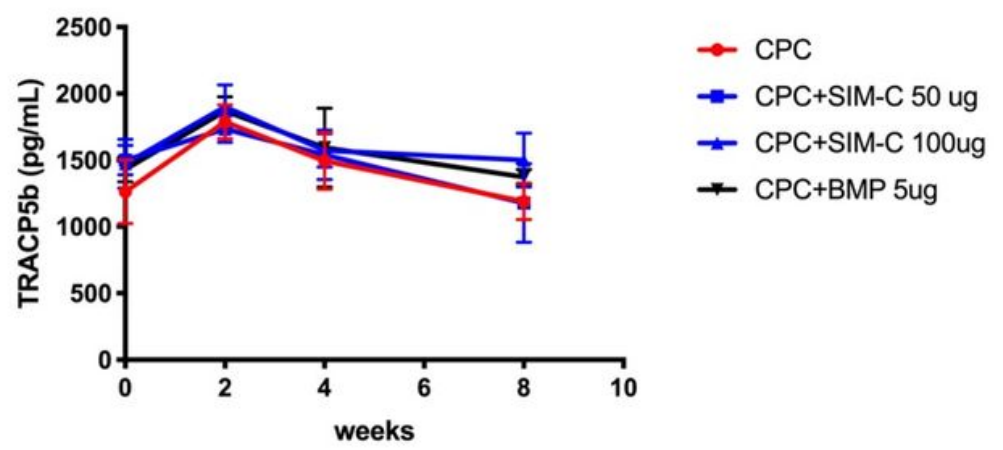

\section{Figure 9}

Serum bone formation marker, intact N-terminal propeptide of type-1 procollagen (P1NP) and bone resorption marker, tartrate-resistant acid phosphatase $5 b$ (TRACP $5 b$ ) in different groups. All data are expressed as mean \pm SD. 